EPJ Web of Conferences 58, 01004 (2013)

DOI: $10.1051 /$ epjconf/20135801004

(C) Owned by the authors, published by EDP Sciences, 2013

\title{
Inextendibilty of the Maximal Global Hyperbolic Development in Electrogowdy spacetimes
}

\author{
Ernesto Nungesser ${ }^{1}$ \\ ${ }^{1}$ Department of Mathematics \\ Royal Institute of Technology \\ Lindstedtsvägen, 25 \\ 10044 Stockholm, Sweden
}

\begin{abstract}
The problem of determinism in General Relativity appears even if one assumes that the spacetime is globally hyperbolic, i.e. that it contains a hypersurface that is intersected by any causal curve exactly once. The strong cosmic censorship hypothesis is essentially the hypothesis that General Relativity is a predictable theory and thus a crucial issue in Classical General Relativity. We sketch here the proof for the case of Electrogowdy spacetimes.
\end{abstract}

\section{Strong cosmic censorship (SCC)}

The first time when in the literature a time machine was decribed, was in 1887 in the novel "El Anacronómete" written by the spaniard Enrique Gaspar [1]. It is clear that a time machine towards the past is related to the problem of the predictability of the theory of General Relativity. It seems that only together with the theory of quantum mechanics there are feasible possibilities at least theoretically for the construction of a time machine, although a lot of difficulties remain. We refer to [2] for recent progress. However even without these remarkable possibilities the question of predictability is still open. It has been shown that the Einstein equations can be seen as an initial value problem [3], [4]. From the singularity theorems of Hawking and Penrose [5],[6],[7] we know that singularities appear under general physically reasonable assumptions, but we do not know what happens in the singularities without a theory of quantum gravity. So if the singularities can influence what is outside, there would be a breakdown of predictability. To prove global existence or the existence of crushing singularities is thus a fundamental question. A conjecture to solve the question of predictability is the strong cosmic censorship hypothesis of Penrose [8]. We will use the following definition of SCC taken from [9]:

Definition 1. SCC The maximal Cauchy development of all initial data for the Einstein-matter system belonging to an open dense subset is inextendible provided the matter model is well-behaved.

This ensures that solutions which are non-generic or "unstable" and with a "badly-behaved" matter model are not taken into account (see chapter 9.4 of [9] for a discussion of that). Since the maximal Cauchy development is the largest region of spacetime which is uniquely determined by initial data, if this region is inextendible, general relativity would remain a deterministic theory. In practice one 
has to look at the structure of the singularities (initial sinuglarity) or to show that there do not exist (in the future for example). Strictly speaking not related to SCC in general, but assuming symmetries one can look what can be shown and often the techniques used can be generalized at least in principle.

\section{Gowdy symmetry}

Gowdy spacetimes are vacuum spacetimes which model closed universes filled with gravitational waves of two polarizations [10]-[11]. We refer to [12] for a detailed mathematical discussion. These spacetimes are a good toy model for the understanding of inhomogeneous and anisotropic cosmological models. See [13], [14], [15] and [16] for recent results on the understanding of inhomogeneous spacetimes near the singularity. Since we will assume some matter content, in particular a Maxwell field, we will use the terminology of Gowdy-symmetric spacetimes instead of Gowdy spacetimes where the Gowdy symmetry is defined as follows:

Definition 2. Gowdy symmetry

1. It is a $T^{2}(U(1) \times U(1))$ symmetry with the group action generated by two commuting spacelike Killing vectors $\left(\frac{\partial}{\partial x}\right.$ and $\left.\frac{\partial}{\partial y}\right)$

2. The transformation which simultaneously maps $x$ to $-x$ and $y$ to $-y$ is an isometry

With this group action the possible spatial topologies are basically $S^{3}, S^{2} \times S^{1}$ and $T^{3}$ and in the following the spatial topology is assumed to be the three-dimensional torus.

A metric with Gowdy symmetry is said to be polarized if the individual transformations mapping $x$ to $-x$ and $y$ to $-y$ are symmetries, which has the physical interpretation that the gravitational waves have only one polarization. We will assume that our spacetime has a polarized Gowdy-symmetry.

\subsection{Polarized Gowdy metric}

It has been shown that the area spanned by the two Killing fields can be used as a time coordinate and moreover that there exists a Cauchy hypersurface of constant $t$. Using this areal time coordinate the polarized Gowdy metric can be put in the following manner:

$$
t^{-\frac{1}{2}} e^{\frac{\lambda(t, \theta)}{2}}\left(-d t^{2}+d \theta^{2}\right)+t\left(e^{P(t, \theta)} d x^{2}+e^{-P(t, \theta)} d y^{2}\right)
$$

Here $\theta, x$ and $y$ are periodic coordinates on $T^{3}$ and $\lambda$ and $P$ are smooth functions.

\section{Einstein-Maxwell equations}

\subsection{Maxwell field and new variables}

The matter content will be described by a Maxwell field which is defined by a four-potential $A_{\alpha}$. We choose a field which is consistent with the symmetries and denote the remaining components of the potential by

$$
\begin{aligned}
& A_{2}=\omega \\
& A_{3}=\chi .
\end{aligned}
$$


Before coming to the equations we introduce new variables

$$
\begin{aligned}
\bar{P} & =\frac{1}{2}(P-\log t) \\
\bar{\lambda} & =\frac{1}{4}(\lambda-\log t)-\bar{P} .
\end{aligned}
$$

What we obtain is the same PDE-system as in the non-polarized Gowdy case! That this choice of variables linking the two cases exists was already known long time ago (see for instance [17]). This enabled us to use the work of Ringström and others obtained for the Gowdy spacetimes which has culminated in [18]. We refer to [19]-[20] for important results concerning SCC in the case of inhomogeneous spacetimes with collisionless matter.

\subsection{Basic equations for Einstein-Maxwell assuming polarized Gowdy symmetry}

The basic equations are the following:

$$
\begin{aligned}
& -\bar{P}_{t t}-t^{-1} \bar{P}_{t}+\bar{P}_{\theta \theta}=e^{2 \bar{P}}\left(-\chi_{t}^{2}+\chi_{\theta}^{2}\right) \\
& -\chi_{t t}-t^{-1} \chi_{t}+\chi_{\theta \theta}=-2\left(-\bar{P}_{t} \chi_{t}+\bar{P}_{\theta} \chi_{\theta}\right) \\
& \bar{\lambda}_{t}=t\left[\bar{P}_{t}^{2}+\bar{P}_{\theta}^{2}+e^{2 \bar{P}}\left(\chi_{t}^{2}+\chi_{\theta}^{2}\right)\right] \\
& \bar{\lambda}_{\theta}=2 t\left[\bar{P}_{t} \bar{P}_{\theta}+e^{2 \bar{P}} \chi_{t} \chi_{\theta}\right]
\end{aligned}
$$

where the first two are the evolution and the last two the constraint equations. There is also a consistency condition which lead us to assume that $\omega=0$ and other equations involving higher derivatives of $\lambda$ which are not important here. Also an integral constraint coming from the fact that $\lambda$ is periodic has to be satisfied. In terms of our new variables the metric is

$$
e^{2(\bar{\lambda}+\bar{P})}\left(-d t^{2}+d \theta^{2}\right)+t^{2} e^{2 \bar{P}} d x^{2}+e^{-2 \bar{P}} d y^{2} .
$$

\section{Central results}

In the following theorems the topology on the set of initial data used is the $C^{\infty}$ topology. $\mathcal{G}_{c}$ is a generic set of initial data satisfying an integral constraint. Data belonging to $\mathcal{G}_{c}$ are called generic. This subset is open and dense in the set of initial data in the $C^{\infty}$ topology.

Theorem 1. There is an open dense subset $\mathcal{G}_{c}$ of the set of smooth initial data for the Einstein-Maxwell equations with polarized Gowdy symmetry and constant areal time such that the Kretschmann scalar tends to infinity along any inextendible past-directed causal geodesic.

Another result is that strong cosmic censorship holds for polarized Electrogowdy, more precisely

Theorem 2. For data belonging to the open dense subset $\mathcal{G}_{c}$ of the set of smooth initial data for the Einstein-Maxwell equations with polarized Gowdy symmetry and constant area radius the corresponding maximal Cauchy development is inextendible.

The proofs and details can be found in [21]. We refer to [22] and [23] for recent results on the foliation of Einstein-Euler spacetimes with Gowdy symmetry which are a first step towards the understanding in the case where the matter source is a fluid. 


\section{References}

[1] E. Gaspar, El Anacronópete (Daniel Cortezo y C, 1887)

[2] S. Finnazi, C. Barceló, S. Liberati, Phys. Rev. D 79, 124017 (2009)

[3] Y. Choquet-Bruhat, R. Geroch, Comm. Math. Phys. 14, 329 (1969)

[4] Y. Fourès-Bruhat, Acta Mathematica 88, 141 (1952)

[5] S.W. Hawking, Proc. Roy. Soc. Lond. A300, 182 (1967)

[6] S.W. Hawking, R. Penrose, Proc. Roy. Soc. Lond. A314, 529 (1970)

[7] R. Penrose, Phys. Rev. Lett. 14, 57 (1965)

[8] R. Penrose, in General Relativity, an Einstein Centenary Survey, edited by S.W. Hawking, W. Israel (Cambridge University Press, Cambridge, UK, 1979)

[9] A.D. Rendall, Partial differential equations in general relativity (Oxford University Press, Oxford, UK, 2008)

[10] R.H. Gowdy, Phys. Rev. Lett. 27, 827 (1971)

[11] R.H. Gowdy, Ann. Phys. (NY) 83, 203 (1974)

[12] P.T. Chruściel, Ann. of Phys. (NY) 202, 100 (1990)

[13] W.C. Lim, Class. Quantum Grav. 25, 045014 (2008)

[14] W.C. Lim, L. Andersson, D. Garfinkle, F. Pretorius, Phys. Rev. D 79, 123526 (2009)

[15] A.A. Coley, W.C. Lim, Phys. Rev. Lett. 108, 191101 (2012)

[16] J.M. Heinzle, C. Uggla, W.C. Lim, Phys. Rev. D 86, 104049 (2012)

[17] M. Carmeli, C. Charach, S. Malin, Survey of cosmological models with gravitational, scalar and electromagnetic waves, Vol. 76, 2 (1981)

[18] H. Ringström, Annals of Mathematics 170, 1181 (2009)

[19] M. Dafermos, A.D. Rendall, arXiv:0610075v1 [gr-qc] (2006)

[20] M. Dafermos, A.D. Rendall, arXiv:0701034v1 [gr-qc] (2007)

[21] E. Nungesser, A.D. Rendall, Class. Quant. Grav. 26, 105019 (2009)

[22] P.G. LeFloch, A.D. Rendall, Arch. Rational Mech. Anal. 201, 841 (2011)

[23] N. Grubic, P. LeFloch, arXiv:1212.1301 [gr-qc] (2012) 